UM-P-93/40, OZ-93/10

\title{
A Possible Way of Connecting The Grassmann Variables And The Number of Generation
}

\author{
Xiao-Gang He \\ Research Center for High Energy Physics \\ School of Physics \\ University of Melbourne \\ Parkville, Vic. 3052 Australia
}

(April, 1993)

\begin{abstract}
We construct a Left-Right symmetric model in which the number of generation is related to Grassmann variables. We introduce two sets of complex Grassmann variables $\left(\theta_{q}^{1}, \theta_{q}^{2}\right),\left(\theta_{l}^{1}, \theta_{l}^{2}\right)$ and associate each variable with leftand right-handed quark and lepton fields, respectively. Expanding quark and lepton fields in powers of the Grassmann variables, we find that there are exactly three generations of quarks and leptons. Integrating out the Grassmann variables, we obtain phenomenologically acceptable fermion mass matrices.
\end{abstract} 11.10.-z, 11.30.Hv, 12.15-y, 12.15.Ff 
How many generations of quarks and leptons are there in the Nature is one of the outstanding problems of particle physics today. Considerations from nuclear synthesis [1] and experimental data on the $\mathrm{Z}$ decay width from LEP [2] both indicate that there are only three generations of light neutrinos, but they do not provide information about the number of heavy generations. On the theoretical side the situation is not any better. The Standard Model does not have the answer to the problem. To answer this question one needs to go beyond the standard model. Many theoretical efforts have been made, ranging from the topological properties of compactified spacetimes in string theory to considerations from anomaly cancellation and composite models, to determine the number of generation [3]. But the problem is still far from been solved. In this letter we will study an interesting approach to the generation problem which relates the particle spectrum with Grassmann variables (GVs) [6. [. terms. This terminating nature of the GVs suggestes a very interesting way to classify particle spectrum when connection between particle fields and the GVs is made. Extensive work has been done with models based on SU(5) grand unification group [5]. In Ref. [5] the particle spectrum, including the gauge transformation properties, are all specified by the GVs. In the follwoing we will study another way of relating the GVs and the number of generation, and to construct a low energy model.

The gauge group of our model is the $S U(3)_{C} \times S U(2)_{L} \times S U(2)_{R} \times U(1)_{B-L}$ Left-Right symmetric group. Under this group the quarks $q$ and leptons $l$ tranform as

$$
\begin{gathered}
q_{L}:(3,2,1,1 / 3), q_{R}:(3,1,2,1 / 3), \\
l_{L}:(1,2,1,-1), l_{R}:(1,1,2,-1) .
\end{gathered}
$$

To make connections between the GVs and the number of generation we introduce two sets GVs: $\theta_{q}=\left(\theta_{q}^{1}, \theta_{q}^{2}\right)$ and $\theta_{l}=\left(\theta_{l}^{1}, \theta_{l}^{2}\right)$ which transform under a global group, $G=$ $S U(2)_{q} \times S U(2)_{l} \times U(1)_{f}$ as $(2,1, \alpha)$ and $(1,2, \alpha)$, respectively. We also group $q_{L, R}$ and $l_{L, R}$ into $Q=\left(q_{L}, q_{R}^{c}\right)$ and $L=\left(l_{L}, l_{R}^{c}\right)$, and let them to transform as $(2,1,0)$ and $(1,2,0)$, respectively. This way of grouping the quarks and leptons suggestes that the global symmetry is in some 
way related to the helicity of fermions. The fermion and boson fields are component fields of some super bosonic fields $E_{i}$ expanded in powers of the GVs. There are two class of expansions of the superfields. One has even powers of the GVs and the other has odd powers. Since the overall superfields $E_{i}$ are bosonic, it is clear that the component fields with even powers of the GVs in the expansion are boson fileds and the ones with odd powers are fermion fields. This expansion does not constrain the gauge transformation properties of the component fields. The superfields can have non-trivial transformation properties under the gauge group.

The Lagrangian density $L$ in the ordinary space-time is obtained by first using the available superfields $E_{i}$ to form terms $L\left(E_{i}\right)$ which are singlets under both the gauge and global symmetries, and then integrating out the GVs, that is

$$
L=\int d^{2} \theta_{q} d^{2} \theta_{l} d^{2} \bar{\theta}_{q} d^{2} \bar{\theta}_{l} L\left(E_{i}\right)
$$

This procedure will select certain terms in $L\left(E_{i}\right)$ because only the terms with proper $\theta$ powers will survive the integral.

Let us consider the fermion fields first. The terms with the lowest power in the GVs for fermion fields are

$$
E_{1 Q}=\bar{\theta}_{q} Q_{1}, E_{1 L}=\bar{\theta}_{l} L_{1}
$$

Multiplying $\left(\bar{\theta}_{q} \theta_{q}\right)^{a}\left(\bar{\theta}_{l} \theta_{l}\right)^{b}$ on $E_{1 i}$, we generate all allowed fermion fields with the same quantum numbers under the global symmetry $\mathrm{G}$ in this theory. We have

$$
\begin{gathered}
E_{1 Q}=\bar{\theta}_{q} Q_{1}, \quad E_{2 Q}=\left(\bar{\theta}_{q} \theta_{q}\right) \bar{\theta}_{q} Q_{2}, \quad E_{3 Q}=\left(\bar{\theta}_{l} \theta_{l}\right) \bar{\theta}_{q} Q_{3}, \\
E_{4 Q}=\left(\bar{\theta}_{l} \theta_{l}\right)^{2} \bar{\theta}_{q} Q_{4}, E_{5 Q}=\left(\bar{\theta}_{q} \theta_{q}\right)\left(\bar{\theta}_{l} \theta_{l}\right) \bar{\theta}_{q} Q_{5}, E_{6 Q}=\left(\bar{\theta}_{q} \theta_{q}\right)\left(\bar{\theta}_{l} \theta_{l}\right)^{2} \bar{\theta}_{q} Q_{6} \\
E_{1 L}=\bar{\theta}_{l} L_{1}, \quad E_{2 L}=\left(\bar{\theta}_{l} \theta_{l}\right) \bar{\theta}_{l} L_{2}, \quad E_{3 L}=\left(\bar{\theta}_{q} \theta_{q}\right) \bar{\theta}_{l} L_{3}, \\
E_{4 L}=\left(\bar{\theta}_{q} \theta_{q}\right)^{2} \bar{\theta}_{l} L_{4}, E_{5 L}=\left(\bar{\theta}_{q} \theta_{q}\right)\left(\bar{\theta}_{l} \theta_{l}\right) \bar{\theta}_{l} L_{5}, E_{6 L}=\left(\bar{\theta}_{l} \theta_{l}\right)\left(\bar{\theta}_{q} \theta_{q}\right)^{2} \bar{\theta}_{l} L_{6} .
\end{gathered}
$$

This set of superfields carries $-\alpha$ of the $U(1)_{f}$ charge. One can generate other fields with different expansions which will have different global symmetry transformation properties. 
Naively, eq.(4) contains six generations of quarks and leptons. This is, however, not true. Some of the fields are actually the same. To see this let us define the dual fields $\tilde{E}_{i}$. The dual of a superfield is defined by replacing the powers of the GVs $\left(\bar{\theta}_{q}\right)^{a}\left(\theta_{q}\right)^{b}\left(\bar{\theta}_{l}\right)^{c}\left(\theta_{l}\right)^{d}$ to $\left(\bar{\theta}_{q}\right)^{2-b}\left(\theta_{q}\right)^{2-a}\left(\bar{\theta}_{l}\right)^{2-d}\left(\theta_{l}\right)^{2-c}$ [4]. We notice that the correct kinetic term in the Lagrangian $L_{k}$ for the fermion fields can be generated by

$$
L_{k}=\int d^{2} \bar{\theta}_{q} d^{2} \bar{\theta}_{l} d^{2} \theta_{q} d^{2} \theta_{l} \bar{E}_{i} \gamma_{\mu} D^{\mu} \tilde{E}_{i}
$$

if the component field of $E_{i}$ is the same as the component filed of its dual. We therefore require that the component field is the same as the component field of its dual. It is easy to see that the following fields are dual pairs

$$
\begin{aligned}
E_{1 Q} \leftrightarrow E_{6 Q}, E_{2 Q} \leftrightarrow E_{4 Q}, E_{3 Q} \leftrightarrow E_{5 Q} . \\
E_{1 L} \leftrightarrow E_{6 L}, E_{2 L} \leftrightarrow E_{4 L}, E_{3 L} \leftrightarrow E_{5 L} .
\end{aligned}
$$

We, therefore, have only three generations of quarks and leptons.

We now turn to possible Higgs scalars $H_{i}$ which may generate fermion masses through Yukawa terms. The Yukawa terms will have the form $E_{i} E_{j} H_{k}$. It is clear that in order to couple the Higgs scalars $H_{i}$ to fermions, $H_{i}$ should carry two times of the $U(1)_{f}$ charge as $E_{i}$ but with opposite signs. We have

$$
\begin{gathered}
H_{1}=\theta_{q} \theta_{q} h_{1}, H_{2}=\bar{\theta}_{l} \theta_{l} \theta_{q} \theta_{q} h_{2}, H_{3}=\left(\bar{\theta}_{l} \theta_{l}\right)^{2} \theta_{q} \theta_{q} h_{3}, \\
H_{4}=\theta_{l} \theta_{l} h_{4}, H_{5}=\bar{\theta}_{q} \theta_{q} \theta_{l} \theta_{l} h_{5}, H_{6}=\left(\bar{\theta}_{q} \theta_{q}\right)^{2} \theta_{l} \theta_{l} h_{6} .
\end{gathered}
$$

From our previous definition for duals, we find $H_{2}$ and $H_{5}$ are self-dual, and $H_{3}, H_{6}$ are the duals of $H_{1}, H_{4}$, respectively. $h_{i}$ are singlets under the global symmetry G. The gauge transformation properties are not specified. In order to form gauge singlets with fermions to generate masses, we assign $h_{i}$ to transform as $(1,2,2,0)$ under the gauge group. Notice that since $h_{i}$ are singlets under the global symmetry G, we can also expaind $H_{i}$ by replacing $h_{i}$ to $\tilde{h}_{i}=\tau_{2} h_{i}^{*} \tau_{2}$ without change the overall transformation properties of $H_{i}$ under the gauge and the global symmetries. Therefore $\tilde{h}_{i}$ should also been included in eq.(7). With the fields in eq.(7), we find that only the following Yukawa terms will survive the GV integration 


$$
\begin{gathered}
E_{1 Q} E_{1 Q} \tilde{H}_{1}, E_{3 Q} E_{3 Q} H_{1}, E_{1 Q} \tilde{E}_{2 Q} H_{1}, E_{1 Q} E_{3 Q} H_{2}, \\
E_{1 L} E_{1 L} \tilde{H}_{4}, E_{3 L} E_{3 L} H_{4}, E_{1 L} \tilde{E}_{2 L} H_{4}, E_{1 L} E_{3 L} H_{5} .
\end{gathered}
$$

Because both $h_{i}$ and $\tilde{h}_{i}$ are available to form gauge singlets with fermions, each term in eq.(8) contains two terms. For quarks we have

$$
E_{i Q} E_{j Q} H_{k} \Rightarrow \lambda_{i j} \bar{q}_{i L} h_{k} q_{j R}+\lambda_{i j}^{\prime} \bar{q}_{i L} \tilde{h}_{k} q_{j R}
$$

where $\lambda$ 's are constants. Similarly for leptons. When $h_{i}$ develop vacuum expectation values, the quarks and leptons obtain their masses. If we now identify

$$
\begin{gathered}
q_{1}=(c, s), q_{2}=(u, d), q_{3}=(t, b), \\
l_{1}=\left(\nu_{\mu}, \mu\right), l_{2}=\left(\nu_{e}, e\right), l_{3}=\left(\nu_{\tau}, \tau\right),
\end{gathered}
$$

we obtain the following form for the fermion mass matrices

$$
M_{f}=\left(\begin{array}{ccc}
0 & a & 0 \\
a^{*} & b & c \\
0 & c^{*} & d
\end{array}\right)
$$

One can have different way to identify the first, the second and the third generations as suggested in eq.(10). However for quarks the identification in eq.(10) is the only one which is consistent with experimental data. For leptons there is more flexibility. In this model neutrinos only have Dirac masses.

In the above discussions only certain superfields are studied. The complete expansion of the superfields in powers of the GVs will generate more particles. There will be several different classes of component fields with different quantum numbers under the global symmetry G. We have no contral of the gauge transformation properties for each class of the fields. However for a given class of the superfields we can follow the some procedure discussed before to determine the number of independent component fields. In this sense the number of generation or particle spectrum is connected with the GVs. 
In conclusion we have suggested a possible way to connect the GVs and the number of generation. We constructed a model with three generations of quarks and leptons and phenomenologically acceptable fermion mass matrices. We must say that more studies are needed to understand the relation betwteen the GVs and the number of generation if they are really connected. The possibility suggested in this paper is only one example.

\section{ACKNOWLEDGMENTS}

I thank Prof. Delbourgo for discussions. This work was supported in part by the Australian Research Council. 


\section{REFERENCES}

[1] See for example, The Early Universe, by E.W. Kolb and M. Turner, Redwood City, USA, Addison-Wesley (1990).

[2] J. Carter, in proceedings of the Joint International Lepton- Photon Symposium and Europhysics Conference on High Energy Physics, Ed. by S. Hegarty, K. Potter and E. Quercigh, Geneva, Switzerland, 25, July - 1, August, 1991.

[3] X.-G. He, G. Joshi, B. McKellar and R. Volkas, Phys. Lett. B 222, 86(1989); R. Peccei, in Proceedings of the Zuoz Spring School, Zuoz, Switzerland, April, 1988, and references therein.

[4] R. Casalbuoni and R. Gatto, Phys. Lett. B88, 306(1979); ibid, B90, 81(1980); W. Krolikowski, Acta. Phys. Polon., B19, 599 (1989).

[5] R. Delbourgo, Mod. Phys. Lett. A4, 1381(1989); R. Delbourgo and M. White, Mod. Phys. Lett. A5, 355(1990); R. Delbourgo, P.D. Jarvis and R.C. Warner, Aust. J. Phys. 44, 135(1991); R. Delbourgo and R. Roleda, Int. J. Mod. Phys. A7, 5213(1992). 